\section{OP9 DO MEALTIME INTERACTIONS, MEALTIME SETTING AND BEDROOM TV SHAPE DIFFERENT TRAJECTORIES OF OVERWEIGHT DURING MIDDLE CHILDHOOD? RESEARCH USING THE GROWING UP IN SCOTLAND STUDY}

AM Parkes*, A Pearce, M Green. MRC/CSO Social and Public Health Sciences Unit, University of Glasgow, Glasgow, UK

\subsection{6/jech-2018-SSMabstracts.9}

Background Potentially modifiable parenting practices relating to mealtime environment and bedroom TV are implicated in the development of overweight and obesity, although it is unknown whether they shape different childhood trajectories of body mass index (BMI), overweight or obesity. The aims of this study were to (1) examine variation in trajectories of overweight among a representative sample of Scottish children; and (2) explore associations with mealtime social interaction and setting, and bedroom TV.

Methods We used the Growing Up in Scotland first birth cohort (baseline in 2004/5, child aged 10 months, $n=5217$ ), selecting families with a singleton birth, with mothers interviewed throughout and with child BMI measurement at 46, 70 , 94 and/or 122 months $(n=2,584)$. Growth mixture modelling (Mplus version 8) identified different trajectories in the likelihood of being overweight $(\geq 85$ th BMI percentile, based on UK growth charts). Multinomial regression models (Stata version 14.2) explored whether informal mealtime setting (away from a dining area, mealtime screen use), positive mealtime interaction ('enjoyable', 'time to talk'), and bedroom TV were associated with overweight trajectories, adjusting for child gender and birthweight and potential confounders (diet quality, permissive feeding, mealtime rules and screen time; maternal BMI; and baseline measures of family socio-economic status, smoking in pregnancy, breastfeeding, and timing of solid feeding).

Results Four overweight trajectories were identified, with sample percentage and probability of being overweight at 46 and 122 months as follows: Low $(58 \%, 0.00,0.10)$, High (13\%, $1.00,0.93)$, Increasing $(19 \%, 0.31,0.84)$ and Decreasing $(10 \%, 0.82,0.00)$. In fully adjusted models, High and Increasing trajectories were more likely than the Low trajectory to have positive mealtime interaction (respective relative risk ratios RRRs with 95\% confidence intervals 1.30 (1.11-1.52), $1.26(1.08-1.47))$; and more likely to have a bedroom TV (1.97 (1.40-2.78), $1.48(1.06-2.05))$. Compared to the Low trajectory, the High trajectory also had a more informal mealtime setting (1.25 (1.09-1.44)). None of the parenting practices distinguished Decreasing from Low or Increasing trajectories. However, compared to the High trajectory, children in the Decreasing trajectory were less likely to have a bedroom TV, informal mealtime setting and positive mealtime interaction: RRRs respectively 0.55 (0.35-0.89), 0.75 (0.630.90), 0.74 (0.60-0.91).

Conclusion Potentially modifiable parenting practices were associated with different trajectories of child overweight. Findings challenge, while not explaining, the role of positive family mealtime interaction in parental management of child overweight, and support likely benefits of reducing screen use at mealtimes and in bedrooms.

\section{OP10 WORSE BASELINE RISK FACTORS EXPLAIN THE ASSOCIATION OF HEALTHY OBESITY WITH INCREASED MORTALITY RISK: WHITEHALL II STUDY}

${ }^{1}$ W Johnson*, ${ }^{2} \mathrm{JA}$ Bell, ${ }^{1} \mathrm{E}$ Robson, ${ }^{1} \mathrm{~T}$ Norris, ${ }^{3} \mathrm{M}$ Kivimaki, 'M Hamer. ${ }^{1}$ School of Sport, Exercise and Health Sciences, Loughborough University, Loughborough, UK; ${ }^{2} M R C$ Integrative Epidemiology Unit, University of Bristol, Bristol, UK; ${ }^{3}$ Department of Epidemiology and Public Health, UCL, London, UK

\subsection{6/jech-2018-SSMabstracts. 10}

Background The extent to which dichotomizing variables to define metabolically healthy obesity (MHO) inherently results in different risk factor trajectories and mortality, relative to a healthy non-obese group, is unknown. We aimed to describe 20-year risk factor trajectories according to initial weight/ health status and investigate the extent to which baseline differences explain greater mortality among MHO than healthy non-obese individuals.

Methods The sample comprised 6529 participants in the Whitehall II study who were measured at five assessments between 1991-1994 and 2012-2013. Baseline weight (nonobese or obese; body mass index (BMI) $\geq 30 \mathrm{~kg} / \mathrm{m}^{2}$ ) and health status (healthy or unhealthy; two or more of hypertension, low high-density lipoprotein cholesterol (HDL-C), high triglycerides, high glucose, and high homeostatic model assessment of insulin resistance (HOMA-IR)) were defined using a standard approach. The relationships of baseline weight/health status with 20-year trajectories summarizing $\sim 25000$ observations of systolic and diastolic blood pressures, HDL-C, triglycerides, glucose, and HOMA-IR were investigated using multilevel models. Associations of baseline weight/health status with all-cause mortality up until July 2015 were investigated using Cox proportional hazards regression. Analyses were adjusted for sex, ethnicity, and baseline covariates (age, alcohol, smoking, exercise, occupational grade, GHQ-rated psychological distress, and diet).

Results Approximately 90\% of the sample was non-obese and $75 \%$ of these individuals were healthy. Conversely, among the $10 \%$ of the sample that was obese only $40 \%$ were healthy. Baseline levels and trajectories of metabolic risk factors tended to be consistently worse for the MHO group compared to the healthy non-obese group (e.g., HOMA-IR by 0.91 (95\% CI $0.67,1.14)$ units at 20 -years of follow-up). Consequently, the MHO group had a greater risk of mortality when the referent group comprised either all healthy non-obese individuals in our sample (hazard ratio $1.57(1.15,2.15)$ ) or a random sample of healthy non-obese individuals (2.11 (1.24, 3.58)). This estimate, however, attenuated $(1.34(0.85,2.13))$ when the referent group was matched to the MHO group on baseline risk factors (i.e., blood pressures, HDL-C, triglycerides, glucose, and HOMA-IR) using propensity scores.

Conclusion This paper demonstrates how dichotomising continuous variables results in different levels of cardio-metabolic disease risk factors over 20 years of follow-up between MHO and healthy non-obese individuals. The greater disease and mortality risk of $\mathrm{MHO}$ compared to healthy non-obese individuals, observed in large scale epidemiological studies, is likely largely explained by the more deleterious risk factor trajectories that result from crude stratification, further challenging the concept of healthy obesity. 